\title{
Generalizations of Lindelöf spaces via hereditary classes
}

\author{
Ahmad Al-omari \\ Al al-Bayt University Faculty of \\ Sciences Department of Mathematics \\ P.O. Box 130095, Mafraq 25113, Jordan \\ email: omarimutah1@yahoo.com
}

\author{
Takashi Noiri \\ 2949-1 Shiokita-cho, Hinagu \\ Yatsushiro-shi, \\ Kumamoto-ken 869-5142 Japan \\ email: t.noiri@nifty.com
}

\begin{abstract}
In this paper by using hereditary classes [6], we define the notion of $\gamma$-Lindelöf modulo hereditary classes called $\gamma \mathcal{H}$-Lindelöf and obtain several properties of $\gamma \mathcal{H}$-Lindelöf spaces.
\end{abstract}

\section{Introduction}

Let $(X, \tau)$ be a topological space and $\mathcal{P}(X)$ the power set of $X$. In 1991, Ogata [13] introduced the notions of $\gamma$-operations and $\gamma$-open sets and investigated the associated topology $\tau_{\gamma}$ and weak separation axioms $\gamma-T_{i}(i=0,1 / 2,1,2)$. In 2011, Noiri [10] defined an operation on an m-structure with property $\mathcal{B}$ (the generalized topology in the sense of Lugojan [8]). The operation is defined as a function $\gamma: \mathrm{m} \rightarrow \mathcal{P}(\mathrm{X})$ such that $\mathrm{U} \subseteq \gamma(\mathrm{U})$ for each $\mathrm{U} \in \mathrm{m}$ and is called a $\gamma$-operation on $m$. Then, it terns out that the operation is an unified form of several operations (for example, semi- $\gamma$-operation [7], pre- $\gamma$-operation [4]) defined on the family of generalized open sets. Moreover, he obtained some characterizations of $\gamma$-compactness and suggested some generalizations of compact spaces by using recent modifications of open sets in a topological space.

2010 Mathematics Subject Classification: 4A05, 54A10

Key words and phrases: $\gamma$-operation, m-structure, m-open, $\gamma$-open, hereditary class, $\mathcal{H}$ Lindelöf, $\gamma \mathcal{H}$-Lindelöf 
In this paper by using hereditary classes [6], we define the notion of $\gamma$ Lindelöf modulo hereditary classes called $\gamma \mathcal{H}$-Lindelöf and obtain several properties of $\gamma \mathcal{H}$-Lindelöf spaces. Recently papers $[1,2,3]$ have introduced some new classes of sets via hereditary classes.

\section{Preliminaries}

First we state the following: in [11], a minimal structure $m$ is defined as follows: $\mathrm{m}$ is called a minima structure if $\emptyset, X \in \mathrm{m}$. However, in this paper, we define as follows:

Definition 1 Let $\mathrm{X}$ be a nonempty set and $\mathcal{P}(\mathrm{X})$ the power set of $\mathrm{X}$. A subfamily $\mathrm{m}$ of $\mathcal{P}(\mathrm{X})$ is called a minimal structure (briefly $\mathrm{m}$-structure) on $\mathrm{X}$ if $\mathrm{m}$ satisfies the following conditions:

1. $\emptyset, x \in m$.

2. The union of any family of subsets belonging to $\mathrm{m}$ belongs to $\mathrm{m}$.

A set $X$ with an $m$-structure is called an $m$-space and denoted by $(X, m)$. Each member of $m$ is said to be $m$-open and the complement of an $m$-open set is said to be m-closed.

Definition 2 [9] Let $\mathrm{X}$ be a nonempty set and $\mathrm{m}$ an $\mathrm{m}$-structure on $\mathrm{X}$. For a subset $\mathrm{A}$ of $\mathrm{X}$, the $\mathrm{m}$-closure of $\mathrm{A}$ is defined as follows: $\operatorname{mcl}(\mathrm{A})=\cap\{\mathrm{F}: \mathrm{A} \subseteq$ $\mathrm{F}, \mathrm{X} \backslash \mathrm{F} \in \mathrm{m}\}$.

Lemma 1 [9] Let $\mathrm{X}$ be a nonempty set and $\mathrm{m}$ an $\mathrm{m}$-structure on $\mathrm{X}$. For the $\mathrm{m}$-closure, the following properties hold, where $\mathrm{A}$ and $\mathrm{B}$ are subsets of $\mathrm{X}$ :

1. $A \subseteq \operatorname{mcl}(A)$,

2. $\operatorname{mcl}(\emptyset)=\emptyset, \operatorname{mcl}(X)=X$,

3. If $\mathrm{A} \subseteq \mathrm{B}$, then $\operatorname{mcl}(\mathrm{A}) \subseteq \operatorname{mcl}(\mathrm{B})$,

4. $\operatorname{mcl}(\operatorname{mcl}(A))=\operatorname{mcl}(A)$.

Lemma 2 [14] Let $(\mathrm{X}, \mathrm{m})$ be an $\mathrm{m}$-space and $\mathrm{A}$ a subset of $\mathrm{X}$. Then $\mathrm{x} \in$ $\operatorname{mcl}(A)$ if and only if $\mathrm{U} \cap \mathrm{A} \neq \emptyset$ for every $\mathrm{U} \in \mathrm{m}$ containing $\mathrm{X}$. 
Lemma 3 [15] Let $(\mathrm{X}, \mathrm{m})$ be an $\mathrm{m}$-space and $\mathrm{A}$ a subset of $\mathrm{X}$. Then, the following properties hold:

1. $A$ is $\mathrm{m}$-closed if and only if $\operatorname{mcl}(\mathrm{A})=\mathrm{A}$,

2. $\operatorname{mcl}(A)$ is $\mathrm{m}$-closed.

Definition 3 [10] Let $(\mathrm{X}, \mathrm{m})$ be an $\mathrm{m}$-space and $\gamma$ an operation on $\mathrm{m}$. A subset $\mathrm{A}$ of $\mathrm{X}$ is said to be $\gamma$-open if for each $\mathrm{x} \in \mathrm{A}$ there exists $\mathrm{U} \in \mathrm{m}$ such that $\mathrm{x} \in \mathrm{U} \subseteq \gamma(\mathrm{U}) \subseteq \mathrm{A}$. The complement of a $\gamma$-open set is said to be $\gamma$-closed. The family of all $\gamma$-open sets of $(\mathrm{X}, \mathrm{m})$ is denoted by $\gamma(\mathrm{X})$.

\section{$3 \quad \gamma \mathcal{H}$-Lindelöf spaces}

First, we recall the definition of a hereditary class used in the sequel. A subfamily $\mathcal{H}$ of the power set $\mathcal{P}(X)$ is called a hereditary class on $X \quad[6]$ if it satisfies the following property: $\mathrm{A} \in \mathcal{H}$ and $\mathrm{B} \subseteq \mathrm{A}$ implies $\mathrm{B} \in \mathcal{H}$.

Definition 4 Let $(\mathrm{X}, \mathrm{m}, \mathcal{H})$ be a hereditary $\mathrm{m}$-space and $\gamma$ an operation on $\mathrm{m}$, where $\mathcal{H}$ is a hereditary class on $\mathrm{X}$. Then $\mathrm{m}$-space $(\mathrm{X}, \mathrm{m})$ is said to be $\gamma \mathcal{H}$ Lindelöf (resp. $\mathcal{H}$-Lindelöf) if every cover $\left\{\mathrm{U}_{\alpha}: \alpha \in \Delta\right\}$ of $\mathrm{X}$ by m-open sets, there exists a countable subset $\Delta_{0}$ of $\Delta$ such that $\mathrm{X} \backslash \cup\left\{\gamma\left(\mathrm{U}_{\alpha}\right): \alpha \in \Delta_{0}\right\} \in \mathcal{H}$ (resp. $\mathrm{X} \backslash \cup\left\{\mathrm{U}_{\alpha}: \alpha \in \Delta_{0}\right\} \in \mathcal{H}$ ).

Theorem 1 Let $(\mathrm{X}, \mathrm{m}, \mathcal{H})$ be a hereditary $\mathrm{m}$-space and $\gamma$ an operation on $\mathrm{m}$, where $\mathcal{H}$ is a hereditary class. Then the following properties are equivalent:

1. $(\mathrm{X}, \gamma(\mathrm{X}))$ is $\mathcal{H}$-Lindelöf;

2. For every family $\left\{\mathrm{F}_{\alpha}: \alpha \in \Delta\right\}$ of $\gamma$-closed sets such that $\cap\left\{\mathrm{F}_{\alpha}: \alpha \in \Delta_{0}\right\} \notin$ $\mathcal{H}$ for every countable subfamily $\Delta_{0}$ of $\Delta, \cap\left\{\mathrm{F}_{\alpha}: \alpha \in \Delta\right\} \neq \emptyset$.

Proof. $(1) \Rightarrow(2)$ : Let $(X, \gamma(X))$ be $\mathcal{H}$-Lindelöf. Suppose that $\cap\left\{F_{\alpha}: \alpha \in\right.$ $\Delta\}=\emptyset$, where $F_{\alpha}$ is $\gamma$-closed set. Then $X \backslash F_{\alpha}$ is $\gamma$-open for each $\alpha \in \Delta$ and $\cup_{\alpha \in \Delta}\left(X \backslash F_{\alpha}\right)=X \backslash \cap_{\alpha \in \Delta}\left(F_{\alpha}\right)=X$. By (1), there exists a countable subfamily $\Delta_{0}$ of $\Delta$ such that $X \backslash \cup_{\alpha \in \Delta_{0}}\left(X \backslash F_{\alpha}\right)=\cap\left\{F_{\alpha}: \alpha \in \Delta_{0}\right\} \in \mathcal{H}$. This is a contradiction.

$(2) \Rightarrow(1)$ : Suppose that $(X, \gamma(X))$ is not $\mathcal{H}$-Lindelöf. There exists a cover $\left\{U_{\alpha}: \alpha \in \Delta\right\}$ of $X$ by $\gamma$-open sets such that $X \backslash \cup\left\{U_{\alpha}: \alpha \in \Delta_{0}\right\} \notin \mathcal{H}$ for 
every countable subset $\Delta_{0}$ of $\Delta$. Since $X \backslash \mathrm{U}_{\alpha}$ is $\gamma$-closed for each $\alpha \in \Delta$ and $\cap\left\{\left(X \backslash \mathrm{U}_{\alpha}\right): \alpha \in \Delta_{0}\right\} \notin \mathcal{H}$ for every countable subset $\Delta_{0}$ of $\Delta$. By (2), we have $\cap\left\{\left(X \backslash \mathrm{U}_{\alpha}\right): \alpha \in \Delta\right\} \neq \emptyset$. Therefore, $\mathrm{X} \backslash \cup\left\{\mathrm{U}_{\alpha}: \alpha \in \Delta\right\} \neq \emptyset$. This is contrary that $\left\{\mathrm{U}_{\alpha}: \alpha \in \Delta\right\}$ is a $\gamma$-open cover of $X$.

Lemma 4 [10] Let $(\mathrm{X}, \mathrm{m})$ be an $\mathrm{m}$-space. For $\gamma(\mathrm{X})$, the following properties hold:

1. $\emptyset, X \in \gamma(X)$,

2. If $A_{\alpha} \in \gamma(X)$ for each $\alpha \in \Lambda$, then $\cup_{\alpha \in \Lambda} A_{\alpha} \in \gamma(X)$,

3. $\gamma(X) \subseteq \mathrm{m}$.

Definition 5 [10] An $\mathrm{m}$-space $(\mathrm{X}, \mathrm{m})$ is said to be $\gamma$-regular if for each $\mathrm{x} \in \mathrm{X}$ and each $\mathrm{U} \in \mathrm{m}$ containing $\mathrm{x}$, there exists $\mathrm{V} \in \mathrm{m}$ such that $\mathrm{x} \in \mathrm{V} \subseteq \gamma(\mathrm{V}) \subseteq \mathrm{U}$.

Lemma 5 [10] For an $\mathrm{m}$-space $(\mathrm{X}, \mathrm{m})$, the following properties are equivalent:

1. $\mathrm{m}=\gamma(\mathrm{X})$;

2. $(\mathrm{X}, \mathrm{m})$ is $\gamma$-regular;

3. For each $\mathrm{x} \in \mathrm{X}$ and each $\mathrm{U} \in \mathrm{m}$ containing $\mathrm{x}$, there exists $\mathrm{W} \in \gamma(\mathrm{X})$ such that $\mathrm{x} \in \mathrm{W} \subseteq \gamma(\mathrm{W}) \subseteq \mathrm{U}$.

Theorem 2 Let $(\mathrm{X}, \mathrm{m}, \mathcal{H})$ be a hereditary $\mathrm{m}$-space and $\gamma$ an operation on $\mathrm{m}$, where $\mathcal{H}$ is a hereditary class. The implications (1) $\Rightarrow(2) \Rightarrow(3) \Rightarrow(4)$ hold. If $(\mathrm{X}, \mathrm{m})$ is $\gamma$-regular, then the following properties are equivalent:

1. $(\mathrm{X}, \mathrm{m})$ is $\mathcal{H}$-Lindelöf;

2. (X, m) is $\gamma \mathcal{H}$-Lindelöf;

3. $(\mathrm{X}, \gamma(\mathrm{X}))$ is $\mathcal{H}$-Lindelöf;

4. $(\mathrm{X}, \gamma(\mathrm{X}))$ is $\gamma \mathcal{H}$-Lindelöf.

Proof. $(1) \Rightarrow(2)$ : Let $(X, m)$ be $\mathcal{H}$-Lindelöf. For any cover $\left\{U_{\alpha}: \alpha \in \Delta\right\}$ of $X$ by m-open sets, there exists a countable subset $\Delta_{0}$ of $\Delta$ such that $X \backslash \cup\{\gamma(U \alpha)$ : $\left.\alpha \in \Delta_{0}\right\} \subseteq X \backslash \cup\left\{U \alpha: \alpha \in \Delta_{0}\right\} \in \mathcal{H}$. Therefore, $(X, m)$ is $\gamma \mathcal{H}$-Lindelöf. 
$(2) \Rightarrow(3)$ : Let $(X, m)$ be $\gamma \mathcal{H}$-Lindelöf and $\left\{\mathrm{U}_{\alpha}: \alpha \in \Delta\right\}$ a cover of $X$ by $\gamma$-open sets. For each $x \in X$ there exists $\alpha(x) \in \Delta$ such that $x \in U_{\alpha(x)}$. Since $\mathrm{U}_{\alpha(\mathrm{x})}$ is $\gamma$-open, there exists $\mathrm{V}_{\alpha(\mathrm{x})} \in \mathrm{m}$ such that $\mathrm{x} \in \mathrm{V}_{\alpha(\mathrm{x})} \subseteq \gamma\left(\mathrm{V}_{\alpha(\mathrm{x})}\right) \subseteq \mathrm{U}_{\alpha(\mathrm{x})}$. Since the family $\left\{V_{\alpha(x)}: x \in X\right\}$ is a cover of $X$ by $m$-open sets and $(X, m)$ is $\gamma \mathcal{H}$-Lindelöf, there exists a countable number of points, say, $x_{1}, x_{2}, x_{3}, \cdots \in X$ such that $X \backslash \cup_{i=1}^{\infty} \gamma\left(V_{\alpha\left(x_{i}\right)}\right) \in \mathcal{H}$ and hence $X \backslash \cup_{i=1}^{\infty} U_{\alpha\left(x_{i}\right)} \in \mathcal{H}$. This shows that $(\mathrm{X}, \gamma(\mathrm{X}))$ is $\mathcal{H}$-Lindelöf.

$(3) \Rightarrow(4)$ : By Lemma $4, \gamma(X)$ is an m-structure and it follows that the same argument as $(1) \Rightarrow(2)$ that $(X, \gamma(X))$ is $\gamma \mathcal{H}$-Lindelöf.

$(4) \Rightarrow(1)$ : Suppose that $(X, m)$ is $\gamma$-regular. Let $(X, \gamma(X))$ be $\gamma \mathcal{H}$-Lindelöf. Let $\left\{U_{\alpha}: \alpha \in \Delta\right\}$ be any cover of $X$ by $m$-open sets. For each $x \in X$, there exists $\alpha(x) \in \Delta$ such that $x \in U_{\alpha(x)}$. Since $(X, m)$ is $\gamma$-regular, by Lemma 5 there exists $\mathrm{V}_{\alpha(\mathrm{x})} \in \gamma(\mathrm{X})$ such that $x \in \mathrm{V}_{\alpha(\mathrm{x})} \subseteq \gamma\left(\mathrm{V}_{\alpha(\mathrm{x})}\right) \subseteq \mathrm{U}_{\alpha(\mathrm{x})}$. Since $\left\{V_{\alpha(x)}: x \in X\right\}$ is a cover of $X$ by $\gamma$-open sets and $(X, \gamma(X))$ is $\gamma \mathcal{H}$-Lindelöf, there exist a countable number of points, say, $x_{1}, \chi_{2}, \chi_{3}, \cdots \in X$ such that $\mathrm{X} \backslash \cup_{i=1}^{\infty} \gamma\left(\mathrm{V}_{\alpha\left(x_{i}\right)}\right) \in \mathcal{H}$; and hence $\mathrm{X} \backslash \cup_{i=1}^{\infty} \mathrm{U}_{\alpha\left(x_{i}\right)} \in \mathcal{H}$. This shows that $(\mathrm{X}, \mathrm{m})$ is $\mathcal{H}$-Lindelöf.

Definition 6 Let $(\mathrm{X}, \mathrm{m}, \mathcal{H})$ be a hereditary $\mathrm{m}$-space. A subset $\mathrm{A}$ of $\mathrm{X}$ is said to be $\gamma \mathcal{H}$-Lindelöf relative to $\mathrm{X}$ if for every cover $\left\{\mathrm{U}_{\alpha}: \alpha \in \Delta\right\}$ of $\mathrm{A}$ by $\mathrm{m}$-open sets of $\mathrm{X}$, there exists a countable subset $\Delta_{0}$ of $\Delta$ such that $A \backslash \cup\left\{\gamma\left(\mathrm{U}_{\alpha}\right): \alpha \in\right.$ $\left.\Delta_{0}\right\} \in \mathcal{H}$.

Theorem 3 Let $(\mathrm{X}, \mathrm{m}, \mathcal{H})$ be a hereditary $\mathrm{m}$-space. If $\mathrm{A}$ is $\gamma$-closed and $\mathrm{B}$ is $\gamma \mathcal{H}$-Lindelöf relative to $\mathrm{X}$, then $\mathrm{A} \cap \mathrm{B}$ is $\gamma \mathcal{H}$-Lindelöf relative to $\mathrm{X}$.

Proof. Let $\left\{V_{\alpha}: \alpha \in \Delta\right\}$ be a cover of $A \cap B$ by m-open subsets of $X$. Then $\left\{V_{\alpha}: \alpha \in \Delta\right\} \cup\{X \backslash A\}$ is a cover of $B$ by $m$-open sets. Since $X \backslash A$ is $\gamma$ open, for each $x \in X \backslash A$, there exists an m-open set $V_{x}$ such that $x \in V_{x} \subseteq$ $\gamma\left(\mathrm{V}_{\mathrm{x}}\right) \subseteq \mathrm{X} \backslash A$. Thus $\left\{\mathrm{V}_{\alpha}: \alpha \in \Delta\right\} \cup\left\{\mathrm{V}_{\mathrm{x}}: \mathrm{x} \in \mathrm{X} \backslash \mathrm{A}\right\}$ is a cover of $\mathrm{B}$ by $\mathrm{m}$ open sets of $X$. Since B is $\gamma \mathcal{H}$-Lindelöf relative to $X$, there exist a countable subset $\Delta_{0}$ of $\Delta$ and a countable points, says $x_{1}, x_{2}, \cdots \in X \backslash A$ such that $\mathrm{B} \subseteq\left[\left(\cup_{\alpha \in \Delta_{0}} \gamma\left(\mathrm{V}_{\alpha}\right)\right) \cup\left(\cup_{i=1}^{\infty} \gamma\left(\mathrm{V}_{x_{i}}\right)\right)\right] \cup \mathrm{H}_{0} \in \mathcal{H}$, where $\mathrm{H}_{0} \in \mathcal{H}$. Hence $\mathrm{A} \cap$ $\mathrm{B} \subseteq\left[\left(\cup_{\alpha \in \Delta_{0}} \gamma\left(\mathrm{V}_{\alpha}\right) \cap A\right) \cup\left(\cup_{i=1}^{\infty} \gamma\left(\mathrm{V}_{x_{i}}\right) \cap A\right)\right] \cup\left(A \cap \mathrm{H}_{0}\right) \subseteq \cup_{\alpha \in \Delta_{0}} \gamma\left(\mathrm{V}_{\alpha}\right) \cup \mathrm{H}_{0}$. Therefore, $A \cap B \backslash\left(\cup_{\alpha \in \Delta_{0}} \gamma\left(V_{\alpha}\right)\right) \subseteq H_{0} \in \mathcal{H}$. Hence $A \cap B$ is $\gamma \mathcal{H}$-Lindelöf relative to $X$.

Corollary 1 If a hereditary $\mathrm{m}$-space $(\mathrm{X}, \mathrm{m}, \mathcal{H})$ is $\gamma \mathcal{H}$-Lindelöf space, then every $\gamma$-closed subset of $\mathrm{X}$ is $\gamma \mathcal{H}$-Lindelöf relative to $\mathrm{X}$. 
Proof. The proof is obvious by Theorem 3 .

Lemma 6 [12] For a hereditary $\mathrm{m}$-space $(\mathrm{X}, \mathrm{m}, \mathcal{H})$, the following properties hold:

1. $\mathrm{m}_{\mathrm{H}}^{*}$ is an $\mathrm{m}$-structure on $\mathrm{X}$ such that $\mathrm{m}_{\mathrm{H}}^{*}$ has property $\mathcal{B}$ and $\mathrm{m} \subseteq \mathrm{m}_{\mathrm{H}}^{*}$.

2. $\beta(\mathrm{m}, \mathcal{H})=\{\mathrm{U} \backslash \mathrm{H}: \mathrm{U} \in \mathrm{m}, \mathrm{H} \in \mathcal{H}\}$ is a basis for $\mathrm{m}_{\mathrm{H}}^{*}$. such that $\mathrm{m} \subseteq$ $\beta(m, \mathcal{H})$.

Theorem 4 Let $(\mathrm{X}, \mathrm{m}, \mathcal{H})$ be a hereditary $\mathrm{m}$-space. Then the following properties hold:

1. If $\left(\mathrm{X}, \mathrm{m}_{\mathrm{H}}^{*}, \mathcal{H}\right)$ is $\mathcal{H}$-Lindelöf, then $(\mathrm{X}, \mathrm{m}, \mathcal{H})$ is $\mathcal{H}$-Lindelöf.

2. If $(\mathrm{X}, \mathrm{m}, \mathcal{H})$ is $\mathcal{H}$-Lindelöf and $\mathcal{H}$ is closed under countable union, then $\left(\mathrm{X}, \mathrm{m}_{\mathrm{H}}^{*}, \mathcal{H}\right)$ is $\mathcal{H}$-Lindelöf.

Proof. (1): The proof follows directly from the fact that every m-closed set is $\mathrm{m}_{\mathrm{H}^{-}}^{*}$-closed.

(2): Suppose that $\mathcal{H}$ is closed under countable union and $X$ is $\mathcal{H}$-Lindelöf. Let $\left\{\mathrm{U}_{\alpha}: \alpha \in \Delta\right\}$ be an $\mathrm{m}_{\mathrm{H}^{-}}^{*}$ open cover of $\mathrm{X}$, then for each $x \in X, x \in \mathrm{U}_{\alpha(\mathrm{x})}$ for some $\alpha(x) \in \Delta$. By Lemma 6 there exist $V_{\alpha(x)} \in \mathrm{m}$ and $\mathrm{H}_{\alpha(\mathrm{x})} \in \mathcal{H}$ such that $x \in \mathrm{V}_{\alpha(x)} \backslash \mathrm{H}_{\alpha(\mathrm{x})} \subseteq \mathrm{U}_{\alpha(\mathrm{x})}$. Since $\left\{\mathrm{V}_{\alpha(\mathrm{x})}: \alpha(\mathrm{x}) \in \Delta\right\}$ is an m-open cover of $X$, there exists a countable subset $\Delta_{0}$ of $\Delta$ such that $X \backslash \cup\left\{\mathrm{V}_{\alpha(\mathrm{x})}: \alpha(\mathrm{x}) \in \Delta_{0}\right\}=\mathrm{H} \in \mathcal{H}$. Since $\mathcal{H}$ is closed under countable union, then $\cup\left\{\mathrm{H}_{\alpha(x)}: \alpha(\mathrm{x}) \in \Delta_{0}\right\} \in \mathcal{H}$. Hence, $\mathrm{H} \cup\left[\cup\left\{\mathrm{H}_{\alpha(\mathrm{x})}: \alpha(\mathrm{x}) \in \Delta_{0}\right\}\right] \in \mathcal{H}$. Observe that $\mathrm{X} \backslash \cup\left\{\mathrm{U}_{\alpha}: \alpha \in \Delta_{0}\right\} \subseteq \mathrm{H} \cup$ $\left[\cup\left\{\mathrm{H}_{\alpha(x)}: \alpha(x) \in \Delta_{0}\right\}\right] \in \mathcal{H}$. By the heredity property of $\mathcal{H}$ we have $X \backslash \cup\left\{\mathrm{U}_{\alpha}:\right.$ $\left.\alpha \in \Delta_{0}\right\} \in \mathcal{H}$ and therefore, $\left(\mathrm{X}, \mathrm{m}_{\mathrm{H}}^{*}, \mathcal{H}\right)$ is $\mathcal{H}$-Lindelöf.

\section{Strongly $\mathcal{H}$-Lindelöf spaces}

Definition 7 A subset $\mathrm{A}$ of a hereditary $\mathrm{m}$-space $(\mathrm{X}, \mathrm{m}, \mathcal{H})$ is said to be:

1. Strongly $\mathcal{H}$-Lindelöf relative to $\mathrm{X}$ if for every family $\left\{\mathrm{V}_{\alpha}: \alpha \in \Delta\right\}$ of m-open sets such that $A \backslash \cup_{\alpha \in \Delta} V_{\alpha} \in \mathcal{H}$, there exists a countable subset $\Delta_{0}$ of $\Delta$ such that $\mathrm{A} \backslash \cup_{\alpha \in \Delta_{0}} \mathrm{~V}_{\alpha} \in \mathcal{H}$. If $\mathrm{A}=\mathrm{X}$, then $(\mathrm{X}, \mathrm{m}, \mathcal{H})$ is said to be Strongly $\mathcal{H}$-Lindelöf. 
2. Strongly $\gamma \mathcal{H}$-Lindelöf relative to $\mathrm{X}$ if for every family $\left\{\mathrm{V}_{\alpha}: \alpha \in \Delta\right\}$ of m-open sets such that $A \backslash \cup_{\alpha \in \Delta} V_{\alpha} \in \mathcal{H}$, there exists a countable subset $\Delta_{0}$ of $\Delta$ such that $\mathrm{A} \backslash \cup_{\alpha \in \Delta_{0}} \gamma\left(\mathrm{V}_{\alpha}\right) \in \mathcal{H}$. If $\mathrm{A}=\mathrm{X}$, then $(\mathrm{X}, \mathrm{m}, \mathcal{H})$ is said to be Strongly $\gamma \mathcal{H}$-Lindelöf.

Theorem 5 Let $(\mathrm{X}, \mathrm{m}, \mathcal{H})$ be a hereditary $\mathrm{m}$-space. Then the following properties hold:

1. If $\left(\mathrm{X}, \mathrm{m}_{\mathrm{H}}^{*}, \mathcal{H}\right)$ is Strongly $\mathcal{H}$-Lindelöf, then $(\mathrm{X}, \mathrm{m}, \mathcal{H})$ is Strongly $\mathcal{H}$-Lindelöf.

2. If $(\mathrm{X}, \mathrm{m}, \mathcal{H})$ is Strongly $\mathcal{H}$-Lindelöf and $\mathcal{H}$ is closed under countable union, then $\left(\mathrm{X}, \mathrm{m}_{\mathrm{H}}^{*}, \mathcal{H}\right)$ is Strongly $\mathcal{H}$-Lindelöf.

Theorem 6 Let $(\mathrm{X}, \mathrm{m}, \mathcal{H})$ be a hereditary $\mathrm{m}$-space. Then the following properties are equivalent:

1. $(\mathrm{X}, \mathrm{m}, \mathcal{H})$ is Strongly $\mathcal{H}$-Lindelöf;

2. If $\left\{\mathrm{F}_{\alpha}: \alpha \in \Delta\right\}$ is a family of $\mathrm{m}$-closed sets such that $\cap\left\{\mathrm{F}_{\alpha}: \alpha \in \Delta\right\} \in \mathcal{H}$, then there exists a countable subfamily $\Delta_{0}$ of $\Delta$ such that $\cap\left\{\mathrm{F}_{\alpha}: \alpha \in\right.$ $\left.\Delta_{0}\right\} \in \mathcal{H}$.

Proof. Suppose that $(X, m, \mathcal{H})$ is Strongly $\mathcal{H}$-Lindelöf. Let $\left\{F_{\alpha}: \alpha \in \Delta\right\}$ be a family of $m$-closed sets such that $\cap\left\{F_{\alpha}: \alpha \in \Delta\right\} \in \mathcal{H}$. Then $\left\{X \backslash F_{\alpha}: \alpha \in \Delta\right\}$ is a family of m-open sets of $X$. Let $H=\cap\left\{F_{\alpha}: \alpha \in \Delta\right\} \in \mathcal{H}$. Let $X \backslash H=$ $X \backslash \cap\left\{F_{\alpha}: \alpha \in \Delta\right\}=\cup\left\{X \backslash F_{\alpha}: \alpha \in \Delta\right\}$. Since $(X, m, \mathcal{H})$ is Strongly $\mathcal{H}$-Lindelöf, there exists a countable $\Delta_{0}$ of $\Delta$ such that $X \backslash \cup\left\{X \backslash F_{\alpha}: \alpha \in \Delta_{0}\right\} \in \mathcal{H}$. This implies that $\cap\left\{\mathrm{F}_{\alpha}: \alpha \in \Delta\right\} \in \mathcal{H}$.

Conversely, let $\left\{V_{\alpha}: \alpha \in \Delta\right\}$ be any family of m-open sets of $X$ such that $X \backslash \cup_{\alpha \in \Delta} V_{\alpha} \in \mathcal{H}$. Then $\left\{X \backslash V_{\alpha}: \alpha \in \Delta\right\}$ is a family of m-closed sets of $X$. By assumption we have $\cap\left\{X \backslash V_{\alpha}: \alpha \in \Delta\right\} \in \mathcal{H}$ and there exists a countable subset $\Delta_{0}$ of $\Delta$ such that $\cap\left\{X \backslash V_{\alpha}: \alpha \in \Delta_{0}\right\} \in \mathcal{H}$. This implies that $X \backslash \cup\left\{V_{\alpha}: \alpha \in\right.$ $\left.\Delta_{0}\right\} \in \mathcal{H}$. This shows that $(X, m, \mathcal{H})$ is Strongly $\mathcal{H}$-Lindelöf.

Definition 8 A subset $\mathrm{A}$ of a hereditary $\mathrm{m}$-space $(\mathrm{X}, \mathrm{m}, \mathcal{H})$ is said to be $\mathrm{mH}_{\mathrm{g}}$ closed if for every $\mathrm{U} \in \mathrm{m}$ with $\mathrm{A} \backslash \mathrm{U} \in \mathcal{H}, \operatorname{mcl}(A) \subseteq \mathrm{U}$.

Proposition 1 Let $(\mathrm{X}, \mathrm{m}, \mathcal{H})$ be a hereditary $\mathrm{m}$-space. If $(\mathrm{X}, \mathrm{m}, \mathcal{H})$ is Strongly $\mathcal{H}$-Lindelöf and $\mathrm{A} \subseteq \mathrm{X}$ is $\mathbf{m H}_{\mathrm{g}}$-closed, then $\mathrm{A}$ is Strongly $\mathcal{H}$-Lindelöf relative to $\mathrm{X}$. 
Proof. Let $\left\{V_{\alpha}: \alpha \in \Delta\right\}$ be a family of m-open subsets of $X$ such that $A \backslash$ $\cup_{\alpha \in \Delta} V_{\alpha} \in \mathcal{H}$. Since $A$ is $m \mathcal{H}_{g}$-closed, $\operatorname{mcl}(A) \subseteq \cup_{\alpha \in \Delta} V_{\alpha}$. Then $(X \backslash \operatorname{mcl}(A)) \cup$ $\left[\cup_{\alpha \in \Delta} V_{\alpha}\right]$ is an m-open cover of $X$ and so $X \backslash\left[(X \backslash \operatorname{mcl}(A)) \cup\left[\cup_{\alpha \in \Delta} V_{\alpha}\right]\right] \in \mathcal{H}$. Since $X$ is Strongly $\mathcal{H}$-Lindelöf, there exists a countable subset $\Delta_{0}$ of $\Delta$ such that $X \backslash\left[(X \backslash \operatorname{mcl}(A)) \cup\left[\cup_{\alpha \in \Delta_{0}} V_{\alpha}\right]\right] \in \mathcal{H} . X \backslash\left[(X \backslash \operatorname{mcl}(A)) \cup\left[\cup_{\alpha \in \Delta_{0}} V_{\alpha}\right]\right]=$ $\operatorname{mcl}(A) \cap\left(X \backslash \cup_{\alpha \in \Delta_{0}} V_{\alpha}\right) \supseteq A \backslash \cup_{\alpha \in \Delta_{0}} V_{\alpha}$. Therefore, $A \backslash \cup_{\alpha \in \Delta_{0}} V_{\alpha} \in \mathcal{H}$. Thus, $A$ is Strongly $\mathcal{H}$-Lindelöf relative to $X$.

Theorem 7 Let $(\mathrm{X}, \mathrm{m}, \mathcal{H})$ be a hereditary $\mathrm{m}$-space. Let $\mathrm{A}$ be an $\mathrm{m} \mathcal{H}_{\mathrm{g}}$-closed set such that $\mathrm{A} \subseteq \mathrm{B} \subseteq \operatorname{mcl}(\mathrm{A})$. Then $\mathrm{A}$ is Strongly $\mathcal{H}$-Lindelöf elative to $\mathrm{X}$ if and only if $\mathrm{B}$ is Strongly $\mathcal{H}$-Lindelöf relative to $\mathrm{X}$.

\section{Proof.}

Suppose that $A$ is Strongly $\mathcal{H}$-Lindelöf elative to $X$ and $\left\{\mathrm{V}_{\alpha}: \alpha \in \Delta\right\}$ is a family of m-open sets of $X$ such that $B \backslash \cup_{\alpha \in \Delta} V_{\alpha} \in \mathcal{H}$. By the heredity property, $A \backslash \cup_{\alpha \in \Delta} V_{\alpha} \in \mathcal{H}$ and $A$ is Strongly $\mathcal{H}$-Lindelöf elative to $X$ and hence there exists a countable subset $\Delta_{0}$ of $\Delta$ such that $A \backslash \cup_{\alpha \in \Delta_{0}} V_{\alpha} \in \mathcal{H}$. Since $A$ is $m \mathcal{H}_{\mathrm{g}}$-closed, $\operatorname{mcl}(A) \subseteq \cup_{\alpha \in \Delta_{0}} \mathrm{~V}_{\alpha}$ and $\operatorname{so} \operatorname{mcl}(A) \backslash \cup_{\alpha \in \Delta_{0}} \mathrm{~V}_{\alpha} \in \mathcal{H}$. This implies that $\mathrm{B} \backslash \cup_{\alpha \in \Delta_{0}} \mathrm{~V}_{\alpha} \in \mathcal{H}$.

Conversely, suppose that $B$ is Strongly $\mathcal{H}$-Lindelöf elative to $X$ and $\left\{V_{\alpha}: \alpha \in\right.$ $\Delta\}$ is a family of m-open subsets of $X$ such that $A \backslash \cup_{\alpha \in \Delta} V_{\alpha} \in \mathcal{H}$. Given that $A$ is $\mathrm{mH}_{\mathrm{g}}$-closed, $\operatorname{mcl}(A) \backslash \cup_{\alpha \in \Delta} \mathrm{V}_{\alpha}=\emptyset \in \mathcal{H}$ and this implies $\mathrm{B} \subseteq \cup_{\alpha \in \Delta} \mathrm{V}_{\alpha} \in \mathcal{H}$. Since $B$ is Strongly $\mathcal{H}$-Lindelöf elative to $X$, there exists a countable subset $\Delta_{0}$ of $\Delta$ such that $\mathrm{B} \backslash \cup_{\alpha \in \Delta_{0}} V_{\alpha} \in \mathcal{H}$. Hence $A \backslash \cup_{\alpha \in \Delta_{0}} V_{\alpha} \in \mathcal{H}$.

\section{$5(\gamma, \delta)$-continuous functions}

Definition 9 Let $(\mathrm{X}, \mathrm{m})$ and $(\mathrm{Y}, \mathrm{n})$ be minimal spaces and $\gamma$ (resp. $\delta)$ be an operation on $\mathrm{m}$ (resp. $\mathrm{n})$. Then a function $\mathrm{f}:(\mathrm{X}, \mathrm{m}) \rightarrow(\mathrm{Y}, \mathrm{n})$ is said to be $(\gamma, \delta)$-continuous if for each $\mathrm{x} \in \mathrm{X}$ and each $\mathrm{V} \in \mathrm{n}$ containing $\mathrm{f}(\mathrm{x})$, there exists $\mathrm{U} \in \mathrm{m}$ containing $\mathrm{x}$ such that $\mathrm{f}(\gamma(\mathrm{U})) \subseteq \delta(\mathrm{V})$.

Lemma 7 Let $\mathrm{f}: \mathrm{X} \rightarrow \mathrm{Y}$ be a function.

1. If $\mathcal{H}$ is a hereditary class on $\mathrm{X}$, then $\mathrm{f}(\mathcal{H})$ is a hereditary class on $\mathrm{Y}$.

2. If $\mathcal{H}$ is a hereditary class on $\mathrm{Y}$, then $\mathrm{f}^{-1}(\mathcal{H})$ is a hereditary class on $\mathrm{X}$.

Proof. (1): This is due to Lemma 3.8 of [5].

(2): Let $A \subseteq f^{-1}(H)$, where $H \in \mathcal{H}$. Then $f(A) \subseteq f\left(f^{-1}(H)\right) \subseteq H$. Hence $f(A) \in \mathcal{H}$ and $A \subseteq f^{-1}(f(A)) \in f^{-1}(\mathcal{H})$ and hence $A \in f^{-1}(\mathcal{H})$. 
Theorem 8 Let $(\mathrm{X}, \mathrm{m})$ and $(\mathrm{Y}, \mathrm{n})$ be minimal spaces and $\gamma$ (resp. $\delta)$ be an operation on $\mathrm{m}$ (resp. $\mathrm{n}$ ) and $\mathcal{H}$ be a hereditary class on $\mathrm{X}$. If $(\mathrm{X}, \mathrm{m}, \mathcal{H})$ is $\gamma \mathcal{H}$-Lindelöf and $\mathrm{f}:(\mathrm{X}, \mathrm{m}, \mathcal{H}) \rightarrow(\mathrm{Y}, \mathrm{n})$ is a $(\gamma, \delta)$-continuous surjection, then $(\mathrm{Y}, \mathrm{n}, \mathrm{f}(\mathcal{H}))$ is $\delta \mathrm{f}(\mathcal{H})$-Lindelöf.

Proof. Let $\left\{V_{\alpha}: \alpha \in \Delta\right\}$ be any cover of $Y$ by $n$-open sets. For each $\chi \in X$, there exists $\alpha(x) \in \Delta$ such that $f(x) \in V_{\alpha(x)}$. Since $f$ is $(\gamma, \delta)$-continuous, there exists $\mathrm{U}_{\alpha(\mathrm{x})} \in \mathrm{m}$ containing $\mathrm{x}$ such that $\mathrm{f}\left(\gamma\left(\mathrm{U}_{\alpha(\mathrm{x})}\right)\right) \subseteq \delta\left(\mathrm{V}_{\alpha(\mathrm{x})}\right)$. Since $\left\{\mathrm{U}_{\alpha(\mathrm{x})}: \mathrm{x} \in \mathrm{X}\right\}$ is a cover of $X$ by $m$-open sets and $(X, m, \mathcal{H})$ is $\gamma \mathcal{H}$-Lindelöf, there exist a countable points $x_{1}, x_{2}, x_{3}, \cdots \in X$ such that $X \backslash \cup_{i=1}^{\infty} \gamma\left(U_{\alpha\left(x_{i}\right)}\right)=H_{0}$, where $\mathrm{H}_{0} \in \mathcal{H}$. Therefore, we have $\mathrm{Y} \subseteq \mathrm{f}\left(\cup_{i=1}^{\infty} \gamma\left(\mathrm{U}_{\alpha\left(x_{i}\right)}\right)\right) \cup \mathrm{f}\left(\mathrm{H}_{0}\right) \subseteq \cup_{i=1}^{\infty} \delta\left(\mathrm{V}_{\alpha\left(x_{i}\right)}\right) \cup$ $\mathrm{f}\left(\mathrm{H}_{0}\right)$. Hence $(\mathrm{Y}, \mathrm{n}, \mathrm{f}(\mathcal{H}))$ is $\delta \mathrm{f}(\mathcal{H})$-Lindelöf.

Definition 10 [11] A function $\mathrm{f}:(\mathrm{X}, \mathrm{m}) \rightarrow(\mathrm{Y}, \mathrm{n})$ is said to be $\mathrm{M}$-closed if for each $\mathrm{m}$-closed set $\mathrm{F}$ of $\mathrm{X}, \mathrm{f}(\mathrm{F})$ is $\mathrm{n}$-closed in $\mathrm{Y}$.

Theorem 9 Let $\mathrm{f}:(\mathrm{X}, \mathrm{m}) \rightarrow(\mathrm{Y}, \mathrm{n}, \mathcal{H})$ be an $\mathrm{M}$-closed surjective function. If for every $\mathrm{y} \in \mathrm{Y}, \mathrm{f}^{-1}(\mathrm{y})$ is Strongly $\mathrm{f}^{-1}(\mathcal{H})$-Lindelöf in $\mathrm{X}$, then $\mathrm{f}^{-1}(\mathrm{~A})$ is Strongly $\mathrm{f}^{-1}(\mathcal{H})$-Lindelöf relative to $\mathrm{X}$ whenever $\mathrm{A}$ is Strongly $\mathcal{H}$-Lindelöf relative to $\mathrm{Y}$ and $\mathrm{A} \backslash \mathrm{U} \in \mathcal{H}$ for every $\mathrm{U} \in \mathrm{n}$.

Proof. Let $\left\{V_{\alpha}: \alpha \in \Delta\right\}$ be a family of m-open subsets of $X$ such that $f^{-1}(A) \backslash$ $\cup\left\{V_{\alpha}: \alpha \in \Delta\right\} \in f^{-1}(\mathcal{H})$. For each $y \in A$ there exists a countable subset $\Delta_{0}(\mathrm{y})$ of $\Delta$ such that $f^{-1}(y) \backslash \cup\left\{V_{\alpha}: \alpha \in \Delta_{0}(y)\right\} \in f^{-1}(\mathcal{H})$. Let $V_{y}=\cup\left\{V_{\alpha}: \alpha \in\right.$ $\left.\Delta_{0}(y)\right\}$. Each $V_{y}$ is an $m$-open set in $(X, m)$ and $f^{-1}(y) \backslash V_{y} \in f^{-1}(\mathcal{H})$.

Now each set $f\left(X-V_{y}\right)$ is $n$-closed in $Y$ and hence, $U(y)=Y-f\left(X-V_{y}\right)$ is n-open in $Y$. Note that $f^{-1}(U(y)) \subseteq V_{y}$. Thus, $\{U(y): y \in A\}$ is a family of $n$-open subsets of $Y$ such that $A \backslash \cup\{U(y): y \in A\} \in \mathcal{H}$. Since $A$ is Strongly $\mathcal{H}$-Lindelöf relative to $Y$, there exists a countable subset $\left\{U\left(y_{i}\right): i \in \mathbb{N}\right\}$ such that $A \backslash \cup\left\{U\left(y_{i}\right): i \in \mathbb{N}\right\} \in \mathcal{H}$ and hence $f^{-1}\left[A \backslash \cup\left\{U\left(y_{i}\right): i \in \mathbb{N}\right\}\right]=f^{-1}(A) \backslash$ $\cup\left\{f^{-1}\left(U\left(y_{i}\right)\right): i \in \mathbb{N}\right\} \in f^{-1}(\mathcal{H})$. Since $f^{-1}(A) \backslash \cup\left\{V_{y_{i}}: i \in \mathbb{N}\right\} \subseteq f^{-1}(A) \backslash$ $\cup\left\{f^{-1}\left(U\left(y_{i}\right)\right): i \in \mathbb{N}\right\}$, then $f^{-1}(A) \backslash \cup\left\{V_{y_{i}}: i \in \mathbb{N}\right\}=f^{-1}(A) \backslash \cup\left\{V_{\alpha}: \alpha \in\right.$ $\left.\Delta_{0}\left(y_{i}\right), i \in \mathbb{N}\right\} \in f^{-1}(\mathcal{H})$. Hence, $f^{-1}(A)$ is Strongly $f^{-1}(\mathcal{H})$-Lindelöf relative to $X$.

A subset $\mathrm{K}$ of an $\mathrm{m}$-space is said to be m-compact [14] if every cover of $\mathrm{K}$ by $m$-open sets of $X$ has a finite subcover.

Theorem 10 Let $\mathrm{f}:(\mathrm{X}, \mathrm{m}) \rightarrow(\mathrm{Y}, \mathrm{n}, \mathcal{H})$ be an $\mathrm{M}$-closed surjective function. If for every $\mathrm{y} \in \mathrm{Y}, \mathrm{f}^{-1}(\mathrm{y})$ is $\mathrm{m}$-compact in $\mathrm{X}$, then $\mathrm{f}^{-1}(\mathrm{~A})$ is $\mathrm{f}^{-1}(\mathcal{H})$-Lindelöf relative to $\mathrm{X}$ whenever $\mathrm{A}$ is $\mathcal{H}$-Lindelöf relative to $\mathrm{Y}$. 
Proof. Let $\left\{\mathrm{V}_{\alpha}: \alpha \in \Delta\right\}$ be a cover of $\mathrm{f}^{-1}(\mathrm{~A})$ by m-open sets of $\mathrm{X}$. For each $y \in A$ there exists a finite subset $\Delta_{0}(y)$ of $\Delta$ such that $f^{-1}(y) \subseteq \cup\left\{V_{\alpha}\right.$ : $\left.\alpha \in \Delta_{0}(y)\right\}$. Let $V_{y}=\cup\left\{V_{\alpha}: \alpha \in \Delta_{0}(y)\right\}$. Each $V_{y}$ is an m-open set in $(X, m)$ and $f^{-1}(y) \subseteq V_{y}$. Since $f$ is $M$-closed, by Theorem 3.1 of [11] there exists an $n$-open set $U_{y}$ containing $y$ such that $f^{-1}\left(U_{y}\right) \subseteq V_{y}$. The collection $\left\{U_{y}: y \in A\right\}$ is a cover of $A$ by n-open sets of $Y$. Hence, there exists a countable subcollection $\left\{U_{y(i)}: i \in \mathbb{N}\right\}$ such that $A \backslash \cup\left\{U_{y(i)}: i \in \mathbb{N}\right\} \in \mathcal{H}$. Then $f^{-1}\left(A \backslash \cup\left\{U_{y(i)}: i \in \mathbb{N}\right\}\right)=f^{-1}(A) \backslash \cup\left\{f^{-1}\left(U_{y(i)}\right): i \in \mathbb{N}\right\} \in f^{-1}(\mathcal{H})$. Since $\left.f^{-1}(A) \backslash \cup\left\{V_{y(i)}: i \in \mathbb{N}\right\} \subseteq f^{-1}(A) \backslash \cup\left\{f^{-1}\left(U_{y(i)}\right): i \in \mathbb{N}\right\}\right)$, then $f^{-1}(A) \backslash \cup\left\{V_{y(i)}:\right.$ $i \in \mathbb{N}\} \in \mathfrak{f}^{-1}(\mathcal{H})$. Thus, $f^{-1}(A)$ is $f^{-1}(\mathcal{H})$-Lindelöf relative to $X$.

\section{References}

[1] A. Al-Omari and T. Noiri, On operators in ideal minimal spaces, Mathematica, 58 (81), No 1-2, (2016), 3-13.

[2] A. Al-Omari and T. Noiri, Operators in minimal spaces with hereditary classes, Mathematica, 61 (84), No 2, (2019), 101-110.

[3] A. Al-Omari and T. Noiri, Properties of $\gamma \mathcal{H}$-compact spaces with hereditary classes, Atti della Accademia Peloritana dei Pericolanti, Classe di Scienze Fisiche, Matematiche e Naturali, 98 No. 2 (2020), A4 [11 pages].

[4] T. V. An, D. X. Cuong and H. Maki, On operation-preopen sets in topological spaces, Sci. Math. Japon., 68 (2008), 11-30.

[5] C. Carpintero, E. Rosas, M. Salas-Brown and J. Sanabria, $\mu$-Compactness with respect to a hereditary class, Bol. Soc. Paran. Mat. (3s), 34(2) (2016), 231-236.

[6] Á. Császár, Modification of generalized topologies via hereditary classes, Acta Math. Hungar., 115 (1-2) (2007), 29-35.

[7] G. Sai Sundara Krishnan, M. Ganster and K. Balachandran, Operation approaches on semi-open sets and applications, Kochi J. Math., 2 (2007), $21-33$.

[8] S. Lugojan, Generalized topology, Stud. Cerc. Mat., 34 (1982), 348-360.

[9] H. Maki, K. C. Rao and A. Nagoor Gani, On generalization semi-open and preopen sets, Pure Appl. Math., 49 (2011), 17-29. 
[10] T. Noiri, A unifed theory for generalizations of compact spaces, Ann. Univ. Sci. Budapest., 54 (2011), 79-96.

[11] T. Noiri and V. Popa, A unified theory of closed functions, Bull. Math. Soc. Sci. Math. Roumanie, 49(97)(4) (2006), 371-382.

[12] T. Noiri and V. Popa, Generalizations of closed sets in minimal spaces with hereditary classes, Ann. Univ. Sci. Budapest., 61 (2018), 69-83.

[13] H. Ogata, Operations on topological spaces and associated topology, Math. Japan., 36 (1991), 175-184.

[14] V. Popa and T. Noiri, On M-continuous functions, An. Univ. "Dunarea de Jos" Galati, Ser. Mat. Fiz. Mec. Teor. (2), 18(23) (2000), 31-41.

[15] V. Popa and T. Noiri, On weakly $(\tau, \mathrm{m})$-continuous functions, Rend. Circ. Mat. Palermo (2), 51 (2002), 295-316. 